Article

\title{
The Dark Sector in the Baryon Phase Transition Cosmology
}

\author{
Frederick J. Mayer
}

Mayer Applied Research, Inc., 1417 Dicken Drive, Ann Arbor, MI 48103, email: fmayer@sysmatrix.net

Version May 31, 2019 submitted to Preprints

Abstract: This brief communication considers and illustrates dark matter and dark energy within the Baryon Phase Transition (BPT) cosmological model as well as some experiments that may confirm (or deny) the validity of the model.

$4 \quad$ Keywords: dark matter; dark energy; Compton composites; tresinos

\section{Introduction}

In my recent paper [1], the strangeness of the Standard Model of Cosmology (SMC) (the $\Lambda$ CDM cosmology) was contrasted with our baryon phase transition (BPT) cosmology [2,3]. Because the SMC has been examined for many years now without either a dark matter particle being detected or a dark energy theory having been clearly identified, it is appropriate that the BPT model should be subjected to further examinations to either confirm (or deny) this model. In the present paper, I hope to begin this effort.

In the BPT, the dark matter particle is composed two separate but bound pieces: a proton and a tresino where the latter is itself a Compton-scale composite particle composed of two electrons and a proton bound together in a balance between electrostatic and spin forces [4]. This composite is illustrated below with electrons in green and a proton in red and shows it to have a net negative charge. In order avoid undue repetition, readers should note that details of the physics in this illustrated paper will be found in the references to our published papers.

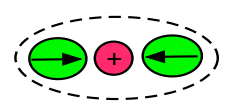

\section{Dark Rotors are the Dark Matter Particles in the BPT Model}

Tresinos are generated about 200 years after the "big bang". Figure 1 illustrates the tresino formation in the early Universe plasma. Note that they are created in pairs along with their recoiling protons.

\section{3. "Free" Tresinos and Protons represent the Dark Energy in the BPT Model}

After the baryon phase transition takes place, the plasma mostly consists of tresinos and protons. But these two components are, of course, attracted to each other so that, in collisions, some are captured as dark rotors as illustrated on the LHS of Figure 2. Those pairs that do not form dark rotors (see [3]) continue on in expansion as "free" tresinos and protons as illustrated in the RHS of Figure 2. These collisions result in about $25 \%$ becoming dark rotors and about $70 \%$ as "free" tresinos and protons. Interestingly, the two latter groups expand much faster than the dark rotors that expand at the speed at which they were created (at a temperature of $\approx 25 \mathrm{eV}$ ) in the Hubble flow (see discussion in [1] and [5]). Note that this result also answers a common cosmological question "where have all the baryons gone?". 


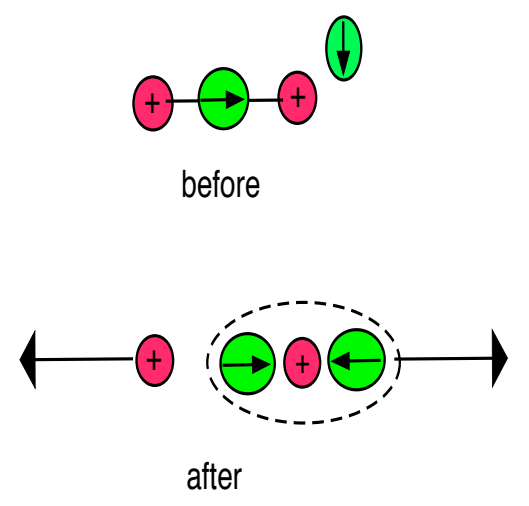

Figure 1. Illustration showing tresino formation and associated proton recoil.
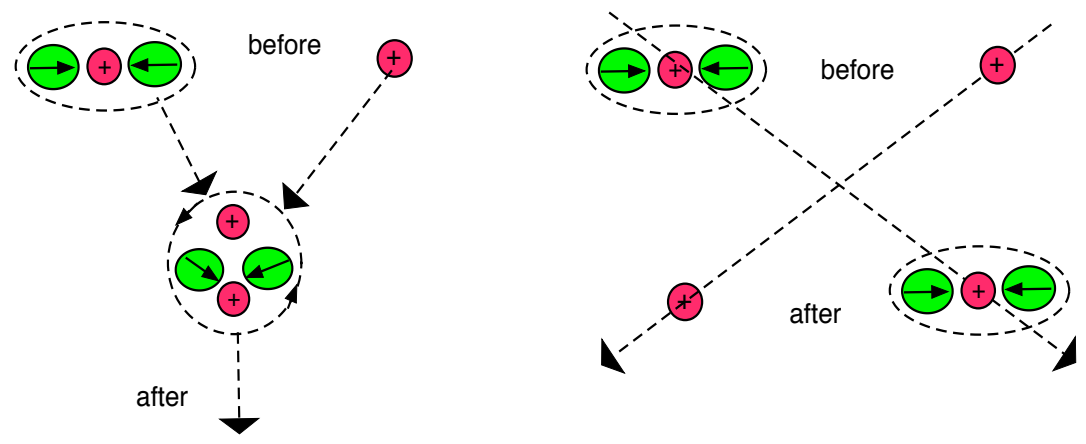

Figure 2. Proton and tresino collisions creating either a dark rotor (LHS) or a "free" proton and "free" tresino (RHS). Dashed circles indicate bound systems.

\section{Dark Rotors in Experiments}

As opposed to the ongoing search for a dark matter particle in the SMC, the BPT's dark rotors have been observed for decades in the so-called $2175 \AA$ A extinction line, as I have discussed in [3]. Below the extinction "fit" is shown Figure 3 and results in the dark rotor density being between 6.7 and $10 \mathrm{~cm}^{-3}$ along this path (taken from [3]).

\section{Rotor Direct-Detection Experiments}

I have previously considered such experiments in [5] and determined that they would be quite difficult to field both because of the inherent size of the rotors and, more importantly, the difficulty of obtaining a "manageable" source of them. However, I hope that this present review will inspire some clever experimentalists to take-up the challenge.

\section{Dark Energy Detection}

As mentioned above, the tresinos and protons (amounting to about 70\% of the initial baryons) that escape becoming dark rotors expand more rapidly than does the Hubble flow, so they are the first mass to populate the late Universe before the formation of the "last scattering surface" and therefore are not registered in the $\mathrm{CMB}$ radiation. These early pairs then represent a charge-neutral background into which the ordinary matter and the dark rotors eventually form and are observed in the CMB, and then become our later Universe. ([1], [5]). 


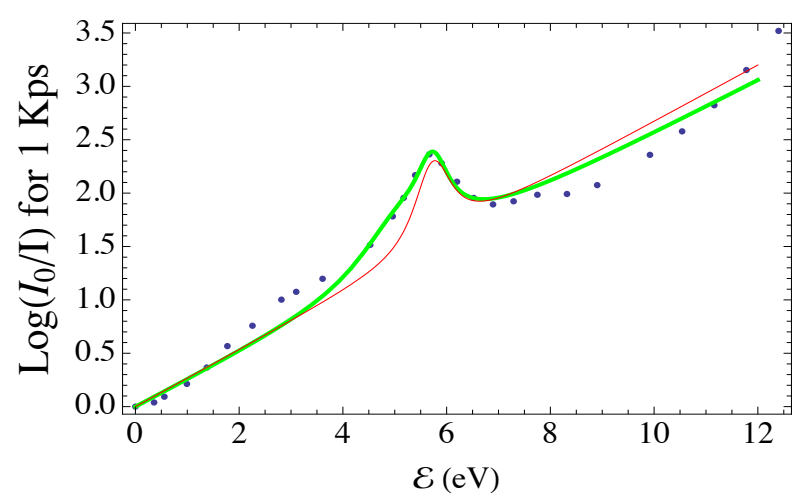

Figure 3. The extinction "fits" obtained from excitations of dark rotors.

$48 \quad 7$. Final Remark

49

53 1. Mayer, F. J., "The Baryon Phase-Transition Model and the too strange Standard Model of Cosmology", Universe $54 \quad 2017,10.3390 /$ universe3010018, 3,18

55 2. Mayer, F. J., \& Reitz, J. R., "Compton Composites Late in the Early Universe", Galaxies 2, 382-409, (2014), 56 doi:10.3390/galaxies2030382, the BPT cosmology.

57 3. Mayer, F. J., "Dark Rotors in the Late Universe", Heliyon 1, (2015) e00039.[FM1]

58 4. Mayer, F. J., Reitz, J. R., “Electromagnetic composites at the Compton scale", Int. J. Theor. Phys. 51 (2012) 322 , 59 arXiv:1110.0034.

60 5. Mayer, F. J., "Hidden Baryons: The Physics of Compton Composites", EPL, 114 (2016) 69001 doi: 61

The BPT cosmology is straightforward as this illustrated version shows. Whether this model is closer to what actually happened in our Universe, or not, will require (as mentioned) some clever experiments to examine the details of its dark sector in future experimental efforts.

10.1209/0295-5075/114/69001. 